\title{
Semaphorin 3F, a chemorepulsant for endothelial cells, induces a poorly vascularized, encapsulated, nonmetastatic tumor phenotype
}

\author{
Diane R. Bielenberg,1,2 Yasuhiro Hida,1,2 Akio Shimizu,1,2 Arja Kaipainen,1,2 Michael Kreuter,3
} Caroline Choi Kim, ${ }^{4}$ and Michael Klagsbrun',2,5

\begin{abstract}
1Vascular Biology Program, and 'Department of Surgery, Children's Hospital, Harvard Medical School, Boston, Massachusetts, USA. ${ }^{3}$ Department of Medicine, Hematology and Oncology, University Hospital of Münster, Münster, Germany. ${ }^{4}$ Department of Dermatology and 5Department of Pathology, Children's Hospital, Harvard Medical School, Boston, Massachusetts, USA.
\end{abstract}

\begin{abstract}
Melanoma is the most lethal skin cancer. Most deaths from melanoma result from metastases. Semaphorins have been shown to inhibit neuronal and endothelial cell migration, but the effects of semaphorins on tumor metastasis have not been documented. We found that semaphorin 3F (SEMA3F) was markedly downregulated in highly metastatic human cell lines in vitro and in vivo, which suggested that it may be a metastasis inhibitor. Metastatic human melanoma cells were transfected with SEMA3F and implanted into mice; the resultant tumors did not metastasize. Rather, the primary tumors resembled benign nevi characterized by large areas of apoptosis, diminished vascularity, inhibition of hyperplasia in overlying epidermal cells, and encapsulated tumor borders delineated by thick layers of fibroblasts and collagen matrix. This phenotype is in stark contrast to highly invasive, vascular mock-transfected tumors. In vitro, tumor cells expressing SEMA3F had a diminished capacity to adhere and migrate on fibronectin. Consistent with semaphorin-mediated chemorepulsion of neurons, tumor cells expressing SEMA3F were chemorepulsive for vascular and lymphatic endothelial cells expressing neuropilin-2 (NRP2), a novel mechanism for a tumor angiogenesis inhibitor. The repulsive activity was abrogated by NRP2 RNA interference. Together these results indicate that SEMA3F is a potent metastasis inhibitor that targets both tumor and stromal cells and raise the possibility of SEMA3F having therapeutic potential.
\end{abstract}

\section{Introduction}

Neuropilins (NRPs) are receptors for both the class 3 semaphorin (SEMA) family of axonal guidance regulators (1) and the VEGF family of angiogenesis factors (2). NRPs are expressed on neurons, endothelial cells (ECs), epithelial cells, osteoblasts, and tumor cells (2-4). There are 2 NRPs, NRP1 and NRP2, which share $44 \%$ amino acid identity and are located on different chromosomes $(5,6)$. In the embryonic vasculature, NRP1 is expressed by vascular ECs whereas NRP2 is expressed by venous ECs and lymphatic ECs $(7,8)$.

The class 3 semaphorins comprise 6 secreted proteins, SEMA3A through SEMA3F. These secreted semaphorins, acting via NRPs, repel axons from growing sensory, sympathetic, and motor neurons $(9,10)$. There is a degree of specificity in semaphorin/NRP binding and activity. NRP1 binds SEMA3A with higher affinity than SEMA3F, whereas NRP2 binds SEMA3F only (5). Functionally, SEMA3A binds NRP1, collapses growth cones on dorsal root

Nonstandard abbreviations used: CRMP, collapsin response mediator protein; DRG, dorsal root ganglia; FN, fibronectin; HPAEC, human pulmonary artery endothelial cell; HUVEC, human umbilical vein endothelial cell; IHC, immunohistochemistry; ISH, in situ hybridization; LEC, lymphatic endothelial cell; LYVE-1, lymphatic vessel endothelial hyaluronan receptor-1; NRP, neuropilin; PAE, porcine aortic endothelial; PCNA, proliferating cell nuclear antigen; RNAi, RNA interference; SCG, superior cervical ganglia; SEMA, semaphorin; SM, A375SM (melanoma cells); SM/Mock, SM (cells) transfected with empty vector only; SM/SEMA3F, SM (cells) expressing SEMA3F.

Conflict of interest: The authors have declared that no conflict of interest exists.

Citation for this article: J. Clin. Invest. 114:1260-1271 (2004).

doi:10.1172/JCI200421378. ganglia (DRG), and repels DRG axons $(1,11)$. SEMA3F binds NRP2 and repels superior cervical ganglia (SCG) $(9,12)$.

NRPs also bind members of the VEGF family. NRP1 binds VEGF-A (165 isoform only), VEGF-B, VEGF-E, and placental growth factor-2, whereas NRP2 binds VEGF-A (145 and 165 isoforms) and VEGF-C (13). NRPs have short cytoplasmic domains with no known signaling motifs. In the neuronal guidance pathway, SEMA3A binds NRP1, and a 3-way complex is formed with plexins, transmembrane receptors that transduce the semaphorin signal (14). In the angiogenesis pathway, NRP1 functions as a coreceptor for VEGFR-2 that increases the binding of VEGF 165 to VEGFR-2 and that enhancesVEGF ${ }_{165}$-mediated chemotaxis of ECs $(2,15)$. Thus, 2 disparate ligand families bind to the same receptor and yet mediate 2 different processes, neuronal guidance and angiogenesis, which suggests common molecular mechanisms for these processes.

NRPs are needed for physiological angiogenesis, as has been shown in mouse embryo knockout studies $(16,17)$ and in zebrafish knockdown studies (18). NRP1 also contributes to tumor vascularization. Overexpression of NRP1 in rat prostate carcinoma cells resulted in tumors that were larger and more vascular $(19,20)$. In contrast, overexpression of soluble NRP1, a secreted protein containing the ligand-binding domain of NRP1, in the same rat prostate cancer cells acted as a tumor antagonist and caused extensive hemorrhage, damaged vessels, and tumor apoptosis (3).

The proangiogenic effects of NRPs have been typically ascribed to their interactions with VEGF $(15,19)$. However, it is now apparent that semaphorins also affect the vasculature, but in an inhibitory 


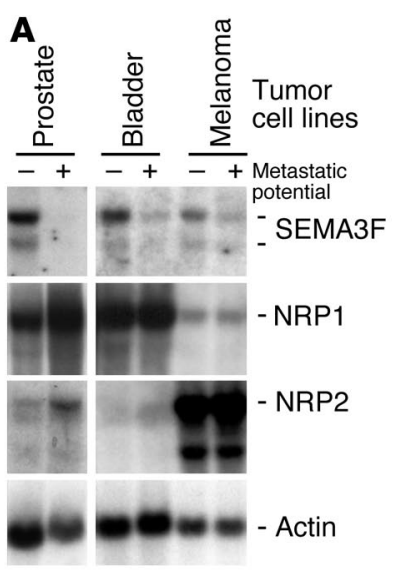

B



C

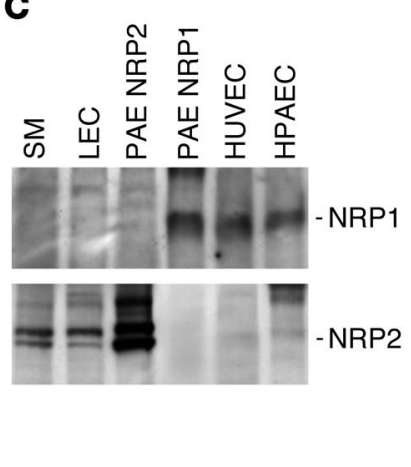

E In vivo

SM Mo H10 A2 A3 C3 D1

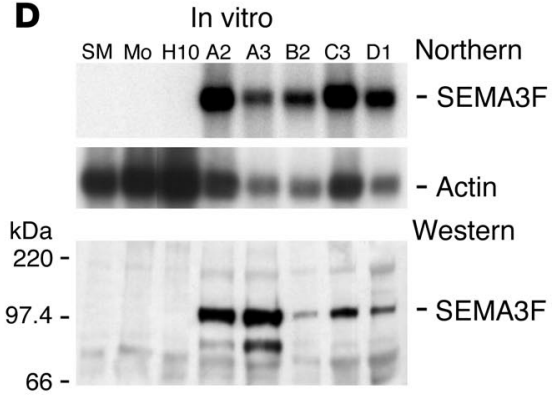

\section{Figure 1}

Metastatic human cell lines downregulate SEMA3F. (A) Northern blot analysis of paired human cancer cell lines with low $(-)$ versus high (+) metastatic potential. The paired cell lines include prostate carcinoma cells PC3M (-) versus PC3MLN4 (+), transitional cell bladder carcinoma cells $253 \mathrm{~J}(-)$ versus 253JBV (+), and melanoma cells A375P $(-)$ versus SM $(+)$. mRNA expression analysis of SEMA3F, NRP1, and NRP2 is demonstrated. (B) RT-PCR analysis for VEGFRs in SM cells and HUVECs. GAPDH was used as a control. (C) Western blot analysis of NRP1 and NRP2 in SM cells and various ECs including LECs, PAE NRP2 cells, PAE NRP1 cells, HUVECs, and HPAECs. (D) Northern blot analysis (top panel) and Western blot analysis (bottom panel) of myc-tagged SEMA3F expression in various transfected clones in vitro. (E) Northern blot analysis of representative tumor clones in vivo. mRNA was isolated directly from tumors grown in mice for 4-5 weeks and blotted for expression of SEMA3F and NRP2. Actin is used as a loading control in A, D (top), and E. SM, (untransfected) A375SM cells; Mo, mock transfected A375SM cells containing empty vector only; $\mathrm{H} 10$, clone transfected with the SEMA3F plasmid that did not express detectable SEMA3F. manner. Previously, we showed that SEMA3A inhibited EC motility in vitro and capillary sprouting from rat aortic ring assays. The inhibition of EC migration was accompanied by rapid disruption of lamellipodia and depolymerization of F-actin in ECs (21). More recently, it has been shown that SEMA3A inhibited EC adhesion and migration in culture (22). In the chick forelimb, SEMA3Acoated beads inhibited blood vessel formation, and SEMA3A antibody caused a local increase in capillary formation (23). SEMA3F has also been shown to inhibit angiogenesis (24).

An important genetic connection between semaphorins and tumor formation was made when SEMA3B and SEMA3F were isolated from a region on human chromosome $3 \mathrm{p} 21.3$ that is commonly deleted in lung cancer $(25,26)$. Based on this correlation, it was suggested that SEMA3B and SEMA3F might be tumor suppressor genes. Transfection of SEMA3B into tumor cells was found to inhibit anchorage-independent growth and tumor formation in HEY ovarian cancer cells and reduce colony formation in vitro by $90 \%$ in NCI-H1299 lung cancer cells $(27,28)$. Overexpression of SEMA3F in the A9 mouse fibrosarcoma cell line or HEY cells inhibited cell growth both in vitro and in vivo but did not affect the growth of lung carcinoma cells NCI-H1299 or GLC45 $(27,29)$. These results suggest that SEMA3B and SEMA3F are functional inhibitors of tumor cell growth.

Whereas previous studies have analyzed semaphorin effects on tumor cell proliferation, effects of SEMA3F on metastasis and tumor phenotype have not been examined. This is an important omission, since most deaths from cancer are due to metastasis. In this report we have examined the inhibitory effects of SEMA3F on metastasis and have explored the mechanisms underlying the inhibition. Our results indicate that the metastatic potential of various human tumor cell lines is correlated with the downregulation of SEMA3F expression. SEMA3F overexpression dramatically altered the invasive, vascular, and metastatic melanoma phenotype to one characterized as benign, necrotic, and encapsulated. Overexpression of SEMA3F also had a direct inhibitory effect on tumor cell adhesion and migration. A novel finding is that tumor cells expressing SEMA3F repelled vascular and lymphatic ECs expressing NRP2 in a manner similar to semaphorin-induced axon repulsion. Here we demonstrate that SEMA3F targets both tumor cells and stromal cells in the tumor microenvironment and potently inhibits metastasis.

\section{Results}

Highly metastatic tumor cells downregulate SEMA3F. Previous genetic studies have implicated SEMA3F as a tumor suppressor $(27,29)$, suggesting that some tumor cells do not express this gene. However, when mRNA expression in various tumor cell lines was analyzed by Northern blot analysis, it was apparent that many tumor cell types express SEMA3F (Figure 1A). Furthermore, when human tumor cell lines with low versus high metastatic potential, e.g., from human prostate carcinoma, bladder carcinoma, and melanoma, were compared, it was found that $S E M A 3 F$ was downregulated in highly metastatic tumor cells (Figure 1A). SEMA3F expression was also absent in metastatic Dunning rat prostate carcinoma cells (AT6.1) as compared with nonmetastatic variants (AT2.1) (data not shown). In contrast to the downregulation of SEMA3F expression in highly metastatic tumor cells, NRP1 and NRP2 were expressed by all of the tumor cell lines analyzed, and there was no consistent correlation between metastatic potential and NRP expression in the paired cell lines.

The highly metastatic A375SM melanoma cells (SM cells) were chosen for further studies because they expressed relatively high levels of NRP2 mRNA (Figure 1, A and B) and protein (Figure 1C). NRP2 is the functional SEMA3F receptor. NRP1 protein was not 


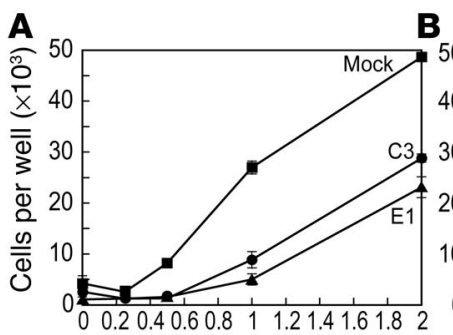

B

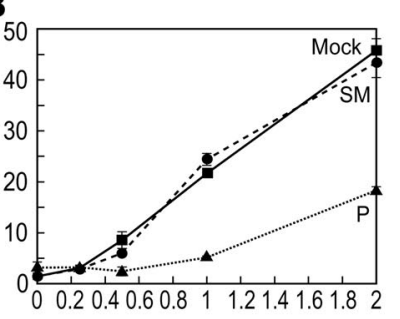

Fibronectin $(\mu \mathrm{g} / \mathrm{ml})$

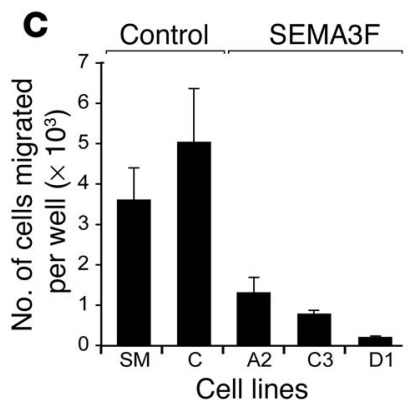

D Fibronectin $(\mu \mathrm{g} / \mathrm{ml})$
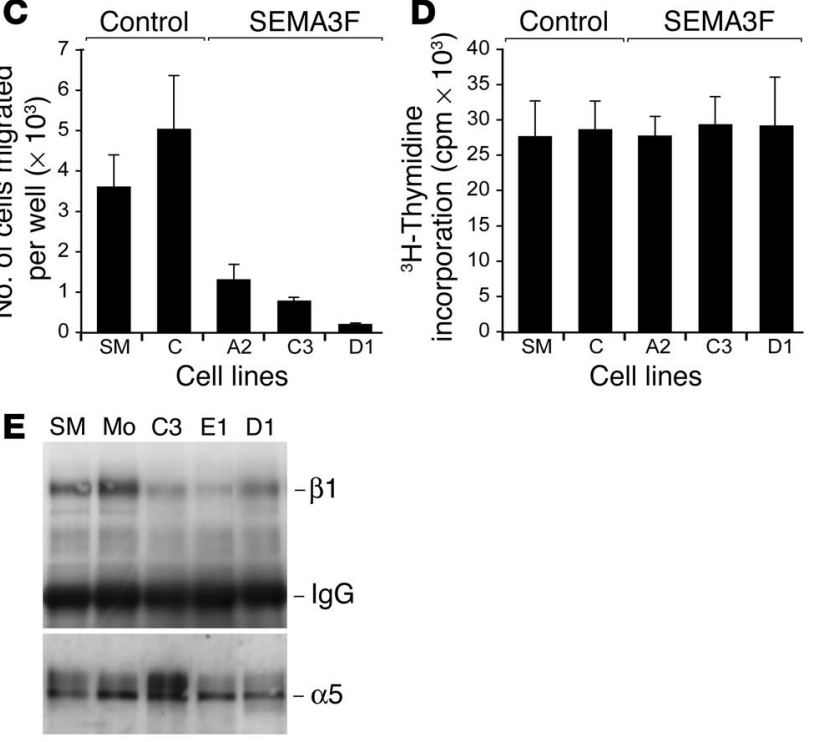

detectable in SM cells (Figure 1C). RT-PCR analysis showed that SM cells did not express VEGFR-1 or VEGFR-2 (Figure 1B). Lack of VEGFR expression was confirmed by Western blot analysis as well as by ${ }^{125}$ I-VEGF cross-linking experiments (data not shown). Together, these results indicate that NRP2 is the only VEGF/semaphorin receptor on SM cells. On the other hand, SM cells secrete VEGF-A ( $3 \mu \mathrm{g} / \mathrm{ml}$, in vitro) as measured by ELISA.

To determine directly whether SEMA3F could inhibit metastasis, the $S E M A 3 F$ gene was transfected into highly metastatic, SM melanoma cells. Zeocin-resistant colonies were selected and expanded. Stable clones A2, A3, B2, C3, and D1 expressed high levels of SEMA3F mRNA by Northern blot analysis (Figure 1D, top) and secreted SEMA3F protein into the media (Figure 1D, bottom). Untransfected SM cells, mock-transfected cells containing empty vector only, and a stable clone transfected with the SEMA3F plasmid (H10) did not express detectable SEMA3F message (Figure $1 \mathrm{D}$, top) or secrete SEMA3F protein (Figure 1D, bottom) and, as a consequence, were used as controls.

The adhesion and migration potential of cells expressing SEMA3F was analyzed in vitro. The adhesion of SM cells expressing SEMA3F (SM/SEMA3F), clones C3 and E1, plated on fibronectin (FN) was decreased fivefold compared with that of mock-transfected controls at $1 \mu \mathrm{g} / \mathrm{ml} \mathrm{FN} \mathrm{(Figure} \mathrm{2A).} \mathrm{The} \mathrm{decrease} \mathrm{in} \mathrm{adhesion} \mathrm{to} \mathrm{FN}$ was not limited to SEMA3F-transfected cell lines but occurred in nonengineered cells as well. Parental nonmetastatic melanoma cells (A375P) expressed abundant SEMA3F, whereas the highly metastatic SM cells did not (Figure 1A). The adhesion of A375P cells to FN was inhibited approximately fivefold compared with the adhesion of SM cells and mock-transfected controls (Figure 2B).

\section{Figure 2}

SEMA3F inhibits adhesion and migration, but not proliferation, of tumor cells. (A) Adhesion of mock-transfected SM cells (squares) and 2 SM/SEMA3F clones, C3 (circles) and E1 (triangles), to FN. (B) Adhesion of parental (A375P) nonmetastatic melanoma cells (triangles), SM metastatic cells (circles), and mock-transfected SM cells (squares) to FN. (C) Boyden chamber motility assay of representative transfected clones. (D) ${ }^{3} \mathrm{H}$-thymidine incorporation into DNA to measure proliferation in representative transfected clones. (E) Western blot analysis of integrins $\beta 1$ and $\alpha 5$ in 3 representative SEMA3F-transfected clones (C3, E1, and D1) following immunoprecipitation with $\alpha 5 \beta 1$ antibody.

Cell motility was measured as the number of cells migrating through an FN-coated membrane in 5 hours. Three different representative SEMA3F-expressing clones (A2, C3, and D1) showed a three- to tenfold diminished basal motility as compared with SM cells or a control clone that did not secrete SEMA3F (Figure 2C). Cell proliferation, as measured by ${ }^{3} \mathrm{H}$-thymidine incorporation (Figure 2D), was not affected by SEMA3F production. Thus, SEMA3F overexpression inhibits melanoma cell adhesion and motility, but not proliferation.

Since tumor cells can interact with ECM via integrins, cell surface molecules that provide connection to adhesive substrates and subsequently signal to the cytoskeleton to enable cell movement, the expression of various integrins involved in adhesion to $\mathrm{FN}$ was investigated. The expression of $\beta 1$ integrin was diminished, as shown by immunoprecipitation and Western blot, in 3 different representative SEMA3F-expressing clones (C3, E1, and D1) as compared with SM and mock-transfected cells (Figure 2E). The protein expression of $\alpha 5$ integrin, the $\alpha$ subunit binding partner of $\beta 1$ in SM cells, did not vary significantly among the clones (Figure $2 \mathrm{E})$. Together these results indicate that SEMA3F is an inhibitor of $\beta 1$ integrin expression and, as a functional consequence, inhibits the adhesion and motility of cells on FN.

Characterization of melanoma cells overexpressing SEMA3F in vivo. $\mathrm{SM} / \mathrm{SEMA3F}$ cells were analyzed for their tumorigenic potential. Various cell lines were injected subdermally into nude mice. Tumorigenicity was $100 \%$ for all of the cell lines examined, and the average diameter of skin tumors appeared similar during the duration of the experiment as measured externally with calipers. Each of the tumors was analyzed for expression of SEMA3F at 4-6 weeks. Northern blot analysis demonstrated that the SM/SEMA3F tumors expressed abundant SEMA3F in vivo whereas the controls did not (Figure 1E). The in vivo expression profiles mimic the in vitro expression profiles shown in Figure 1D. SEMA3F mRNA expression patterns were visualized in all experimental tumors by in situ hybridization (ISH). SEMA3F ISH is shown in 3 representative tumors, 1 control SM tumor (Figure $3 \mathrm{~A}$ ) and 2 SM/SEMA3F tumors (dark purple in Figure 3, B and C). SEMA3F mRNA expression was prominent in transfected tumor cells, whereas stromal cells did not express SEMA3F. Control SM tumors did not express any detectable SEMA3F by ISH (Figure 3A); this confirms in vivo the original observation in Figure $1 \mathrm{~A}$ that metastatic melanoma cells downregulate SEMA3F.

Although overall tumor diameter varied only slightly among the groups, it was apparent from $\mathrm{H} \& \mathrm{E}$ staining that tumors expressing SEMA3F contained large areas void of viable tumor cells (arrows in Figure 3, E and F). These areas were composed of massive numbers of TUNEL-positive apoptotic cells (arrows in Figure 3, H and I) and lacked dividing cells (arrows in Figure 3, K and L). Control tumors, 
SEMA3F expression
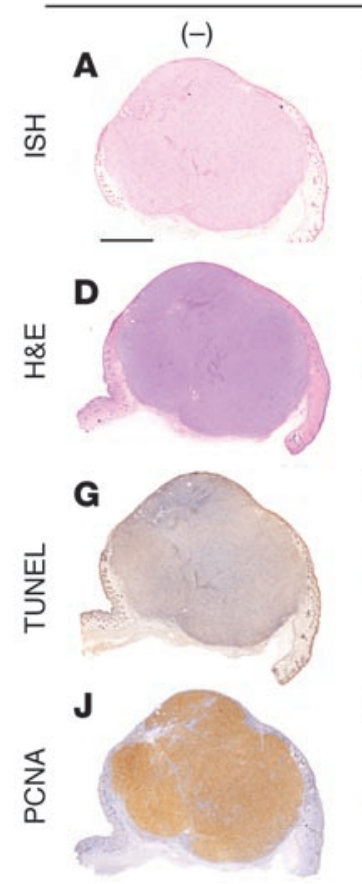

(+) $\mathrm{A} 2$
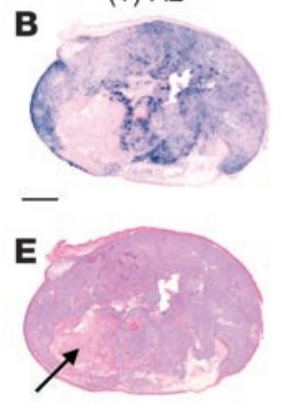

H
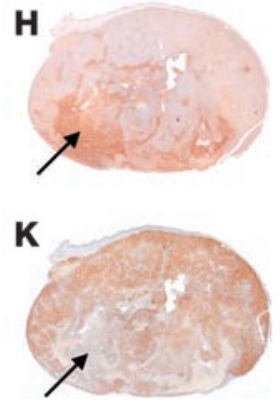

$(+) \mathrm{D} 1$
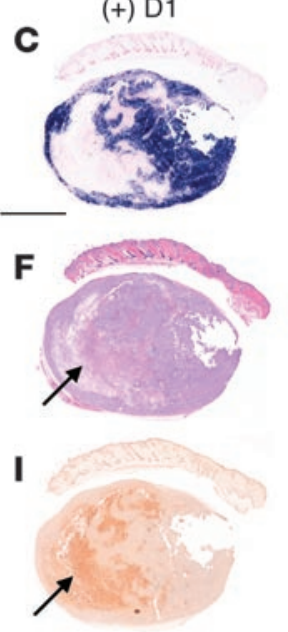

L



Figure 3

SEMA3F overexpression results in tumor apoptosis. Scans of whole-tumor cross sections. (A-C) ISH for SEMA3F expression (dark purple). (D-F) H\&E staining. (G-I) TUNEL staining to detect apoptotic cells (brown). (J-L) PCNA staining to detect dividing cells (brown). Arrows denote areas of apoptosis that lack SEMA3F or PCNA staining. Scale bars: $2 \mathrm{~mm}$.

were detected in the lungs of mice with tumors expressing SEMA3F by H\&E or S100 $\beta$ staining (Figure $4 \mathrm{I}$ ). On the other hand, histological analysis confirmed the presence of macroand micrometastases in the lungs of mice with SEMA3Fnegative tumors (Figure 4, E-H). In another approach, after 12 weeks, lung tissues were dissected, dispersed into culture, and allowed to grow, since growth of melanoma tumor cells in vitro would be evidence of pre-existing metastases in the lungs. Melanoma tumor cells (S100ß positive) grew from lung tissue excised from mice bearing control tumors. However, no tumor cells and only occasional fibroblast colonies were cultured from the lungs of mice bearing tumors expressing SEMA3F (data not shown).

As another method specifically aimed at detecting occult metastases, SM cells, mock-transfected cells, and SEMA3Ftransfected cells (clone A3) were infected with a retrovirus containing GFP. Mice were injected with GFP cells, and tumors were removed after 4 weeks. All primary tumors strongly fluoresced green under a fluorescent dissecting scope and stained positive for GFP using immunohistochemistry (IHC) (not shown). To analyze metastasis, mice were autopsied after 8 more weeks (12 weeks total). Obvious lung metastases were seen readily by green fluorescence (Figure 4J) in mice with control tumors (6 of on the other hand, contained mainly actively dividing cells as identified by proliferating cell nuclear antigen (PCNA) staining (Figure 3J) and were nearly void of apoptotic cells (Figure 3, D and G).

SEMA3F expression inhibits lymph node and lung metastasis. SEMA3F expression in numerous clones completely inhibited metastasis to both lymph nodes and lungs (Figure 4; Table 1). Inguinal lymph node metastases were evident in 22 of 22 mice injected with control clones. However, no lymph node metastases were detected in mice with clones expressing SEMA3F (0 of 27 mice). For an in-depth analysis, right inguinal lymph nodes (closest to tumor implanted on the right dorsal flank) were sectioned throughout and stained by H\&E or immunostained for the melanoma marker S100 $\beta$. Careful histological examination of lymph nodes confirmed the existence of large metastases (nearly the entire node) in mice with control tumors (Figure 4, A and B), but no evidence of metastatic cells was found in the nodes of mice with tumors expressing SEMA3F (Figure 4, C and D).

SEMA3F also inhibited metastasis to the lung (Figure 4; Table 1). Six weeks after tumor cell injection (protocol 1), metastatic tumor nodules were visible on the surface of the lungs in control mice (4 of 4 mice), whereas no lung metastases ( 0 of 8 mice) were detected when tumors expressed SEMA3F. When mice were maintained for 12 weeks following injection (protocol 2), those with prior SEMA3F tumors still had no lung metastases (0 of 19), whereas 17 of 18 mice with prior control tumors had lung metastases (Table 1). Lung tissues from each mouse were sectioned at multiple levels, and no metastases
Table 1

SEMA3F inhibits metastasis to lymph nodes and lung

\begin{tabular}{|c|c|c|c|c|}
\hline $\begin{array}{l}\text { Spontaneous } \\
\text { metastasis }\end{array}$ & $\begin{array}{l}\text { SEMA3F } \\
\text { protein }\end{array}$ & Cell line & $\begin{array}{l}\text { Incidence of lymph } \\
\text { node metastasis }\end{array}$ & $\begin{array}{c}\text { Incidence of } \\
\text { lung metastasis }\end{array}$ \\
\hline Protocol $1^{\mathrm{A}}$ & $\begin{array}{l}- \\
+ \\
+\end{array}$ & $\begin{array}{c}\text { SM } \\
\text { SEMA3F (A2) } \\
\underline{\text { SEMA3F (D1) }}\end{array}$ & $\begin{array}{l}4 / 4 \\
\underline{0 / 4} \\
\underline{0 / 4} \\
\end{array}$ & $\begin{array}{l}4 / 4 \\
\underline{0 / 4} \\
\underline{0 / 4} \\
\end{array}$ \\
\hline Protocol $2^{\mathrm{B}}$ & $\begin{array}{l}- \\
- \\
+ \\
+ \\
+ \\
+ \\
- \\
- \\
+\end{array}$ & $\begin{array}{c}\text { SM } \\
\text { Control (mock) } \\
\text { SEMA3F (A3) } \\
\text { SEMA3F (B2) } \\
\text { SEMA3F (C3) } \\
\text { SEMA3F (D1) } \\
\text { SM/GFP } \\
\text { Mock/GFP } \\
\text { SEMA3F (A3)/GFP }\end{array}$ & $\begin{array}{l}4 / 4 \\
8 / 8 \\
0 / 4 \\
0 / 4 \\
0 / 4 \\
0 / 4 \\
3 / 3 \\
3 / 3 \\
0 / 3 \\
\end{array}$ & $\begin{array}{l}4 / 4 \\
7 / 8 \\
0 / 4 \\
0 / 4 \\
0 / 4 \\
0 / 4 \\
3 / 3 \\
3 / 3 \\
0 / 3 \\
\end{array}$ \\
\hline $\begin{array}{l}\text { Protocols } 1 \text { and } 2 \\
\text { combined }\end{array}$ & $\begin{array}{l}- \\
+\end{array}$ & $\begin{array}{l}\text { Controls } \\
\text { SEMA3F }\end{array}$ & $\begin{array}{l}22 / 22 \\
0 / 27\end{array}$ & $\begin{array}{l}21 / 22 \\
\underline{0 / 27}\end{array}$ \\
\hline $\begin{array}{r}\text { Experimental } \\
\text { metastasis }\end{array}$ & $\begin{array}{l}- \\
- \\
+ \\
+\end{array}$ & $\begin{array}{c}\text { SM } \\
\text { Control (mock) } \\
\text { SEMA3F (C3) } \\
\text { SEMA3F (B2) }\end{array}$ & & $\begin{array}{l}5 / 5^{C} \\
5 / 5^{C} \\
\underline{0 / 5} \\
1 / 5^{D}\end{array}$ \\
\hline
\end{tabular}

SM/SEMA3F cells are underlined; control cells are in regular font. AMice were autopsied at 6 weeks after tumor cell injection. ${ }^{B}$ Subdermal tumors were removed after 4 weeks; mice were autopsied after 12 weeks. CEach mouse had more than 50 metastases. DOne mouse had an encapsulated, avascular micrometastasis, less than $200 \mu \mathrm{m}$ in diameter. 


\section{Lymph nodes}


$(-)$
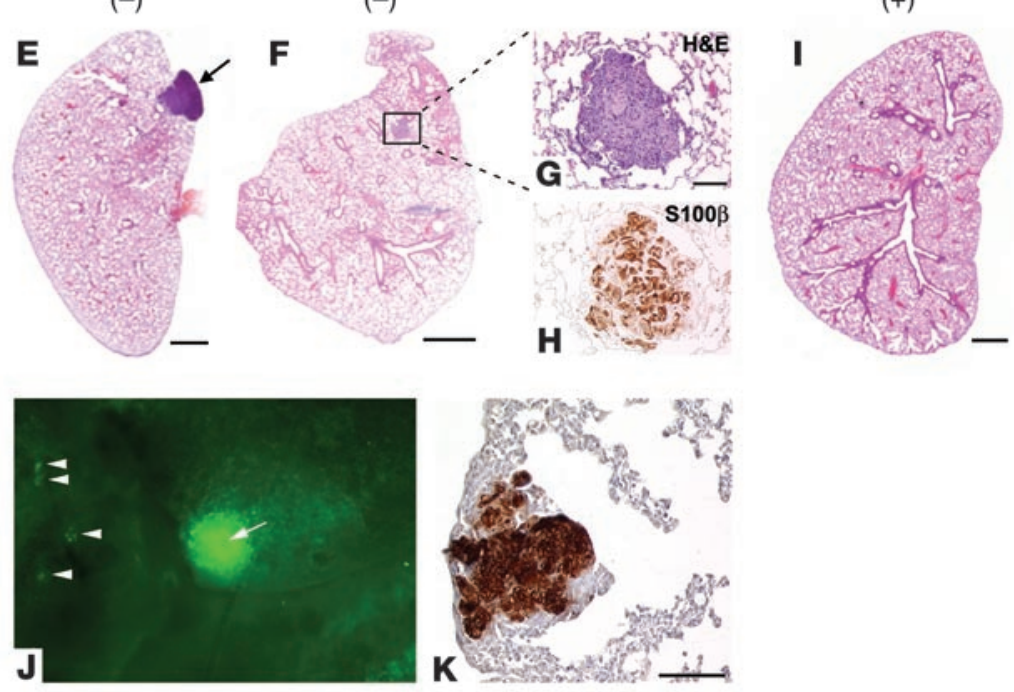

GFP Fluorescence

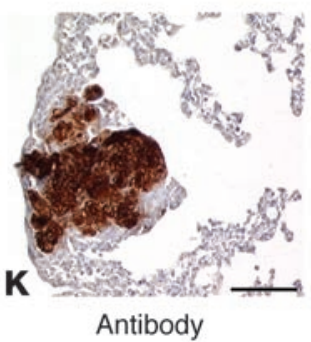

6) but not in mice with tumors expressing SEMA3F (0 of 3) (Table 1). In control mice, GFP-positive micrometastases were detected even in inner lung tissues by IHC (Figure 4K), but again, no GFPpositive metastases of any size were detected in SEMA3F mice.

Experimental metastasis via tail vein injection into the circulation was also carried out (Table 1). After 12 weeks, more than 50 metastases were detected per mouse $(n=10)$ when SM or mocktransfected cells were injected i.v., consistent with previous reports for this cell line (30). However, only 1 very small ( $<200 \mu \mathrm{m})$, avascular, encapsulated metastasis was detected in 1 of 10 mice injected i.v. with SM/SEMA3F cells. These results suggest that SEMA3F can also inhibit lung colonization. From these exhaustive analyses, it was concluded that SEMA3F is a novel potent inhibitor of both lymph node and lung metastasis.

SEMA3F induces a benign tumor phenotype. To determine the mechanisms by which SEMA3F inhibits metastasis, we investigated the primary tumors more carefully and found dramatic differences in the tumor microenvironment of tumors lacking, compared with tumors expressing, SEMA3F. Control SM tumors induced massive keratinocyte hyperplasia in the epidermis overlying the tumors (Figure 5A, bracket). The thickness of the epidermis in a normal mouse skin is typically $2-3$ cell layers, approximately $20 \mu \mathrm{m}$, whereas the epidermis overlying some SM tumors was increased sixfold, approximately $120 \mu \mathrm{m}$ (Figure 5A, bracket). On the other

\section{Figure 4}

SEMA3F inhibits lymph node and lung metastasis. (A-D) Scans of lymph nodes stained with H\&E (A and $\mathbf{C}$ ) or the melanoma marker S100 $\beta$ (B and D, brown). Scale bars: 1 $\mathrm{mm}$. (E, F, and I) Scans of lung sections stained by H\&E. Scale bars: $1 \mathrm{~mm}$. The arrow points to a macrometastasis $(\mathbf{E})$, and the box surrounds a micrometastasis (F). ( $\mathbf{G}$ and $\mathbf{H})$ The micrometastasis in $\mathbf{F}$ is shown magnified and stained with $H \& E(G)$ and $S 100 \beta(H$, brown). Scale bar: $100 \mu \mathrm{m}$. No metastases were detected in lungs of mice bearing SM/SEMA3F tumors (I). ( $\mathbf{J}$ and $\mathbf{K}$ ) Use of GFPinfected cells to detect lung metastases. Large metastasis ( $\mathbf{J}$, arrow) as well as small metastases ( $\mathbf{J}$, arrowheads) fluoresced green in lungs from mice with control tumors. IHC with antibodies to GFP also detected micrometastases in lungs from mice with control tumors (K, brown). Scale bar: $100 \mu \mathrm{m}$.

hand, tumors secreting SEMA3F induced very modest keratinocyte proliferation in the overlying epidermis (Figure 5B, bracket). Another distinctive feature in tumor morphology was that tumors expressing SEMA3F were highly encapsulated with well-defined tumor borders (Figure 5D, bracket) composed of thick layers of fibroblasts and collagen matrix (Figure 5, F and H, brackets), whereas SM tumors were nonencapsulated (Figure 5, C, E, and G, brackets). Taken together, these results suggest that SEMA3F secretion by tumors induces a benign, collagen-rich, encapsulated tumor phenotype.

SEMA3F expression inhibits tumor angiogenesis. Melanoma tumor cells metastasize by disseminating from the primary tumor via blood vessels and lymphatic vessels $(31,32)$. We previously demonstrated that another semaphorin family member, SEMA3A, inhibited endothelial sprouting in an aortic ring assay (21). We hypothesized that SEMA3F may be an inhibitor of angiogenesis as well. In order to analyze the tumor vasculature, cryosections $(8 \mu \mathrm{m})$ were stained by IHC with an antibody to murine CD31 (Figure 6, A-H). CD31 localization was analyzed in entire primary-tumor cross sections (Figure 6, A and B) as well as in representative high-magnification fields from each tumor (Figure 6, C and D). The blood vessels in tumors expressing SEMA3F (Figure 6D) appeared to be smaller, less branched, and fewer in number than control tumor blood vessels (Figure 6C). Quantification of tumor angiogenesis was accomplished by comparison of 100 independent fields from each group using IPLab software (Scanalytics Inc.). Tumors expressing SEMA3F were found to contain nearly half the number of vessels per unit area compared with control tumors $\left(28 / \mathrm{mm}^{2}\right.$ vs. $\left.53 / \mathrm{mm}^{2}\right)$. In addition, SEMA3F expression resulted in diminished average vessel area in tumors to $56 \%$ of control $\left(335 \mu \mathrm{m}^{2}\right.$ vs. $\left.593 \mu \mathrm{m}^{2}\right)$ and diminished total vessel area in tumors to $39 \%$ of control $\left(14,232 \mu \mathrm{m}^{2}\right.$ vs. $\left.36,174 \mu \mathrm{m}^{2}\right)$.

Blood vessel sprouting from the surrounding skin microenvironment into the tumor was analyzed by staining of thick cryosections $(30 \mu \mathrm{m})$ with CD31 antibodies. Control tumors were well vascularized, as was the overlying dermis (Figure 6E), and vessels could be seen to sprout from the underlying dermal blood vessels into control tumors (Figure 6G, asterisks). On the other hand, tumors expressing SEMA3F contained fewer vessels and sprouts with relatively thin EC lining, while most blood vessels remained 
$(-)$


\section{Figure 5}

SEMA3F expression alters the tumor microenvironment. (A and B) H\&E staining of tumors with and without SEMA3F expression and overlying skin. E, epidermis; D, dermis; T, tumor. Scale bar: $100 \mu \mathrm{m}$. Mouse epidermal cells overlying control tumors were hyperplastic (A, bracket), while the epidermis overlying tumors expressing SEMA3F remained at nearly normal thickness (B, bracket). (C-H) Tumor/stromal borders, with and without SEMA3F expression. (C and D) H\&E. (E and F) IHC using antibodies to fibroblasts (brown). ( $\mathbf{G}$ and $\mathbf{H}$ ) Trichrome staining; collagen is stained in blue. (C, E, and $\mathbf{G}$ ) Tumors without SEMA3F had nondelineated borders and invasive tumor leading edges. (D, F, and H) Tumors expressing SEMA3F were surrounded by thick layers of stromal cells (brackets) forming a capsule around the tumor. The capsule was composed largely of fibroblasts $(\mathbf{F})$ that deposited collagen matrix (H). Scale bar: $100 \mu \mathrm{m}$.

peritumoral (Figure 6, F and H). In Figure 6F, it appears that normal skin blood vessels are blocked from invading the tumor.

$S E M A 3 F$ induces EC chemorepulsion in vitro. The blocking of tumor vascularization shown in Figure 6F suggests a possible mechanism whereby SEMA3F repels skin blood vessels in a manner similar to that in which SEMA3F repels growing axons from SCG (12). To investigate this possibility, a coculture system of ECs and tumor cells expressing SEMA3F was established (Figure 7). Porcine aortic endothelial (PAE) cells were used, since they are devoid of endogenous NRP1 and NRP2 and can be stably transfected with either of these genes. PAE cells express plexins A1 and A2, transmembrane receptors that mediate semaphorin signaling, as observed by RTPCR analysis (data not shown). When PAE cells expressing NRP2 were grown to confluence and SM/SEMA3F cells were added in coculture (Figure 7A), large zones of clearance were apparent in the EC culture by 12 hours (Figure 7B). By 48 hours, these zones were much larger (Figure 7C). In the coculture, tumor cells are identi- fied as the larger cells located in the center of the clearance zones (Figure 7, B and C, green arrows). As controls, PAE NRP2 cells did not have zones of clearance when cocultured with tumor cells lacking SEMA3F expression (Figure 7D); similarly, PAE cells expressing NRP1, a functional receptor for SEMA3A, did not retract from SM/SEMA3F cells (Figure 7E; Figure 8C). At 9 hours, multiple zones of clearance can be clearly seen throughout the coculture at lower magnifications (Figure 7, F and G). At higher magnifications (Figure 7, H and I), it is readily apparent that the PAE NRP2 cells segregate away from SM/SEMA3F cells. In these and the following experiments (Figure 7, F-M; Figure 8, A-C and E-G), the SM cells transfected with empty vector only (SM/Mock) or SM/SEMA3F cells, which are melanoma cells, are readily distinguished from ECs by expression of the melanoma-specific marker S100 $\beta$ (brown).

$\mathrm{SM} / \mathrm{SEMA3F}$ cells induced chemorepulsion in nonengineered ECs as well. Besides in PAE NRP2 cells (Figure 7, F-I), zones of clearance were observed in cocultures of SM/SEMA3F cells with human pulmonary artery ECs (HPAECs) (Figure 7J) and human umbilical vein ECs (HUVECs) (Figure 8E). Lymphatic ECs (LECs) express NRP2, and not NRP1 $(8,33)$; thus LECs may be targets of SEMA3F. Indeed, in coculture, zones of clearance were found in the LEC monolayers (Figure 7L). SM cells lacking SEMA3F expression (SM/Mock) did not induce the repulsion of primary HUVECs (Figure 8G), HPAECs (Figure 7K), or LECs (Figure 7M) in any cultures.

Time-lapse photography (Figure $7 \mathrm{~N}$ ) and video (see supplemental data; available at http://www.jci.org/cgi/content/full/114/9/1260/ DC1) show the effects of cocultures dynamically. The panels show a tumor cell expressing SEMA3F at the center (green arrow) and surrounding PAE NRP2 cells retracting from the source of ligand and creating a clearance zone that enlarges with time. The video clearly depicts the repulsion as an active process in which the ECs extend and retract numerous filopodia toward the direction of the SEMA3F ligand while at the same time the cells as a population are moving away from the ligand. One frame of the video (at $\sim 6$ hours) is shown at higher magnification (Figure $7 \mathrm{~N}$, right panel) to point out the numerous EC extensions or filopodia (black arrows) present during the retraction process.

SEMA3F repels PAE NRP2 cells and HUVECs (Figure 8, A and E). To determine whether the SEMA3F-induced repulsion was dependent on NRP2, we used RNAi to silence NRP2. Western blot analysis and immunocytochemistry show that NRP2 RNAi strongly reduces NRP2 protein levels in PAE NRP2 cells (Figure 8, B and D) and in HUVECs (Figure 8H). In addition, immunocytochemical staining for NRP2 (pink) shows that NRP2 RNAi reduces NRP2 protein levels in nearly all cells in the culture (Figure 8, compare A with B). Concomitantly, the repulsive effects of SEMA3F on PAE NRP2 cells (Figure 8B) and HUVECs (Figure 8F) were strongly inhibited by RNAi. As with PAE NRP2/RNA interference (NRP2/ RNAi) cells, no repulsion occurred with PAE NRP1 cells (Figure 8, compare B with $\mathrm{C}$ ). From these results, we conclude that NRP2 is the functional receptor for SEMA3F-induced repulsion of ECs.

\section{Discussion}

Semaphorins, well characterized as regulators of neuronal guidance, have also been implicated as antagonists of tumorigenesis. The secreted, class 3 semaphorins SEMA3B and SEMA3F were first identified in regions of chromosome 3 p21 commonly deleted in lung cancer, which suggested that they had tumor suppressor activity $(25,26,34)$. Further studies showed that SEMA3B and SEMA3F transfected into certain tumor cell lines could inhibit 
tumor cell proliferation in cell culture, and tumor formation in vivo, although not always consistently $(27,29)$. However, these studies did not address the role of semaphorins in tumor invasion, angiogenesis, and metastasis, nor did they address the mechanisms underlying these inhibitory activities. In our initial studies with melanoma, prostate, and bladder tumor cells, we found that benign tumor cells expressed SEMA3F, whereas highly metastatic tumor cells did not, suggesting an inverse relationship between metastatic potential and SEMA3F expression.

In order to determine directly whether SEMA3F could influence the metastatic potential of a tumor cell line, SEMA3F was overexpressed in a highly metastatic human melanoma cell line. SM cells were used for several reasons: (a) of any cell line examined, SM cells express the highest levels of NRP2, the functional receptor of SEMA3F; (b) SM cells constitute an excellent human tumor model system in nude mice, because they can be injected orthotopically into the skin with $100 \%$ tumor take, and tumor volume can be measured externally and (c) SM cells metastasize spontaneously and reproducibly to regional lymph nodes and lungs in nude mice. Large metastases can be seen visually, and even though these melanomas are amelanotic, the disseminated micrometastases can be detected by histological staining with melanoma-specific antibodies such as S100 $\beta$ or HMB45 (35). A thorough analysis was performed to look for metastases, including sectioning of the tissues at multiple levels, staining by standard H\&E, staining with specific melanoma markers, and culturing of minced lung pieces to observe any melanoma cell outgrowth. Controls included nontransfected SM cells and mock-transfected SM cells. Metastasis was analyzed after 6 weeks, and none was detected in lymph nodes and lungs in mice bearing tumors expressing SEMA3F by any of these methods. To address the possibility that the tumor cells did disseminate but were too small to be detected, a protocol was developed to search for occult micrometastases. Even after 12 weeks, none of the 19 mice that had received tumors expressing SEMA3F in protocol 2 had any spontaneous metastases, whereas 17 of 18 mice receiving control tumors had distant lung metastases as determined by H\&E, melanoma marker staining, GFP fluorescence, and melanoma cell outgrowth.

When experimental metastasis was carried out by direct injection of cells into the circulation, obviating the need for tumor cells to migrate to and invade blood vessels, control cells readily metastasized to lung as expected. However, SM/SEMA3F cells did not metastasize, with the exception of 1 micrometastatic lesion (<200 $\mu \mathrm{m})$ in 1 of 10 mice. Thus, SEMA3F inhibits not only migration and invasion at the primary site but lung colonization as well.

What are the mechanisms of SEMA3F-induced inhibition of metastasis? Metastasis is a multistep process (36). In order to metastasize, tumor cells must be able to grow initially at the primary site, migrate and invade blood vessels or lymphatic vessels, survive in the circulation, evade host immune cells, extravasate into the distant organ, and respond to the new organ environment (36). Blocking any 1 step in this process may be enough to inhibit cells from reaching their final destination. One possible mechanism is that SEMA3F acts directly on tumor cells expressing NRP2 to inhibit their tumor activities. A piece of evidence for direct action is that SEMA3F overexpression inhibits basal SM tumor cell migration substantially, three- to tenfold, in a Boyden chamber assay. Furthermore, the adhesion of these cells plated on FN is inhibited fivefold compared with that of controls not expressing SEMA3F. In addition, the adhesion of parental (A375P) nonmetastatic melanoma cells (which express SEMA3F) to
FN is inhibited to a degree similar to that seen in the transfected cells, about fivefold, as compared with the adhesion of the aggressive SM cells (which do not express SEMA3F). The decrease in adhesiveness was correlated with a concomitant decrease in the levels of $\beta 1$ integrin in the SM/SEMA3F cells. The integrins $\alpha 3 \beta 1, \alpha 4 \beta 1$, $\alpha 5 \beta 1$, and $\alpha v \beta 1$ all bind FN; and SM cells express predominantly $\alpha 5 \beta 1$. These results are consistent with earlier studies showing that SEMA3 proteins inhibit $\beta 1$ integrin-mediated adhesion and migration of tumor cells and ECs $(22,37)$. Together, these results suggest that inhibition of tumor metastasis by SEMA3F is correlated with a
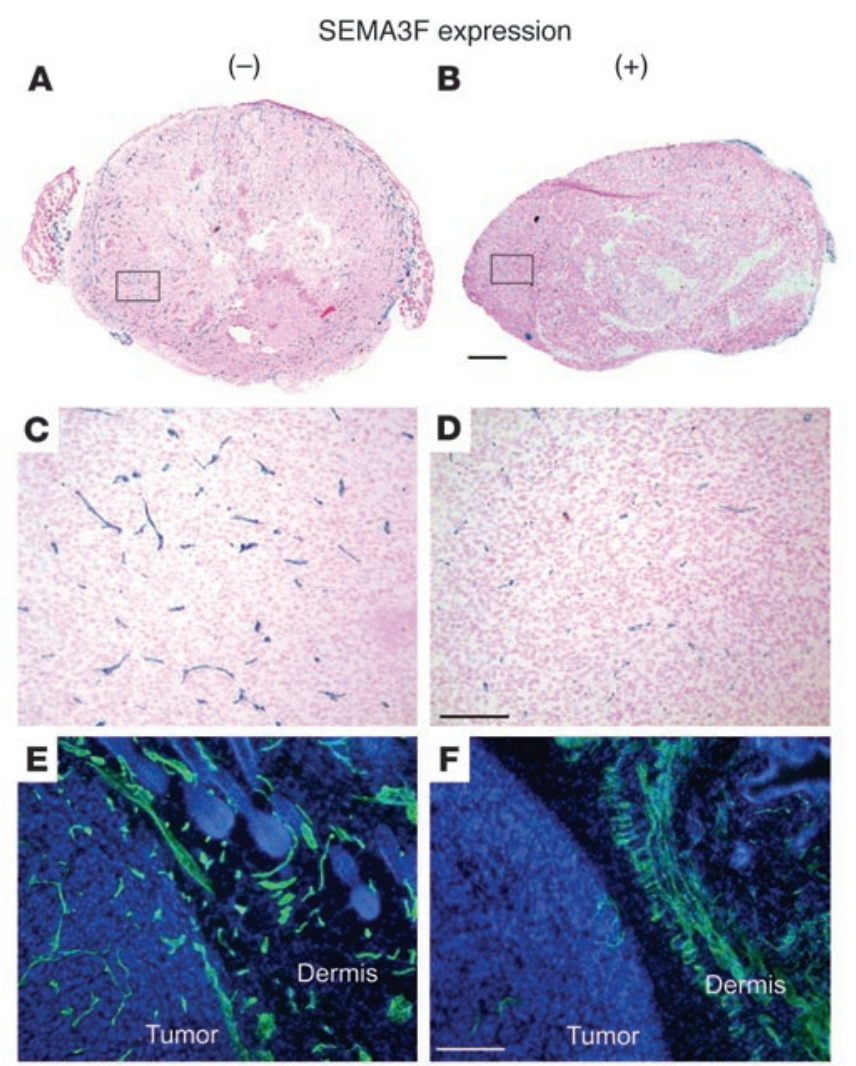

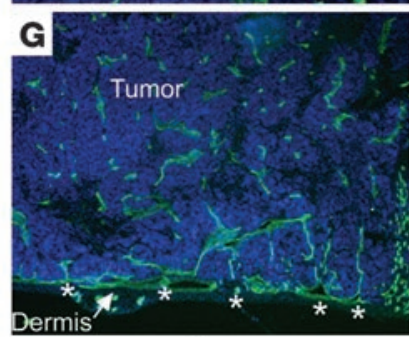

$(-)$

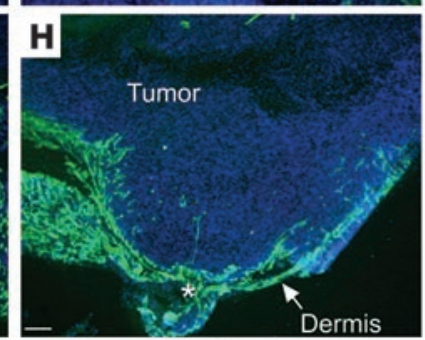

$(+)$

\section{Figure 6}

SEMA3F inhibits tumor angiogenesis. CD31 staining in tumors lacking $(\mathbf{A}, \mathbf{C}, \mathbf{E}$, and $\mathbf{G})$ and expressing $\operatorname{SEMA3F}(\mathbf{B}, \mathbf{D}, \mathbf{F}$, and $\mathbf{H})$. Vessels appear blue in thin $(8 \mu \mathrm{m})$ cryosections $(\mathbf{A}-\mathbf{D})$ or fluorescent green in thick $(30 \mu \mathrm{m})$ cryosections $(\mathbf{E}-\mathbf{H})$. (A and B) Scans of wholeprimary-tumor cross sections stained with CD31. Scale bar: $1 \mathrm{~mm}$. (C and $\mathbf{D}$ ) A representative field from each tumor (in a non-necrotic area) is shown at higher magnification (C from box in $\mathbf{A}$; $\mathbf{D}$ from box in $\mathbf{B}$ ). $(\mathbf{E}-\mathbf{H})$ Thick cryosections were used to detect neovascularization and sprouting (asterisks) from surrounding skin vessels into the tumors. Scale bars in $\mathbf{C}-\mathbf{H}: \mathbf{2} 200 \mathrm{~m}$. 

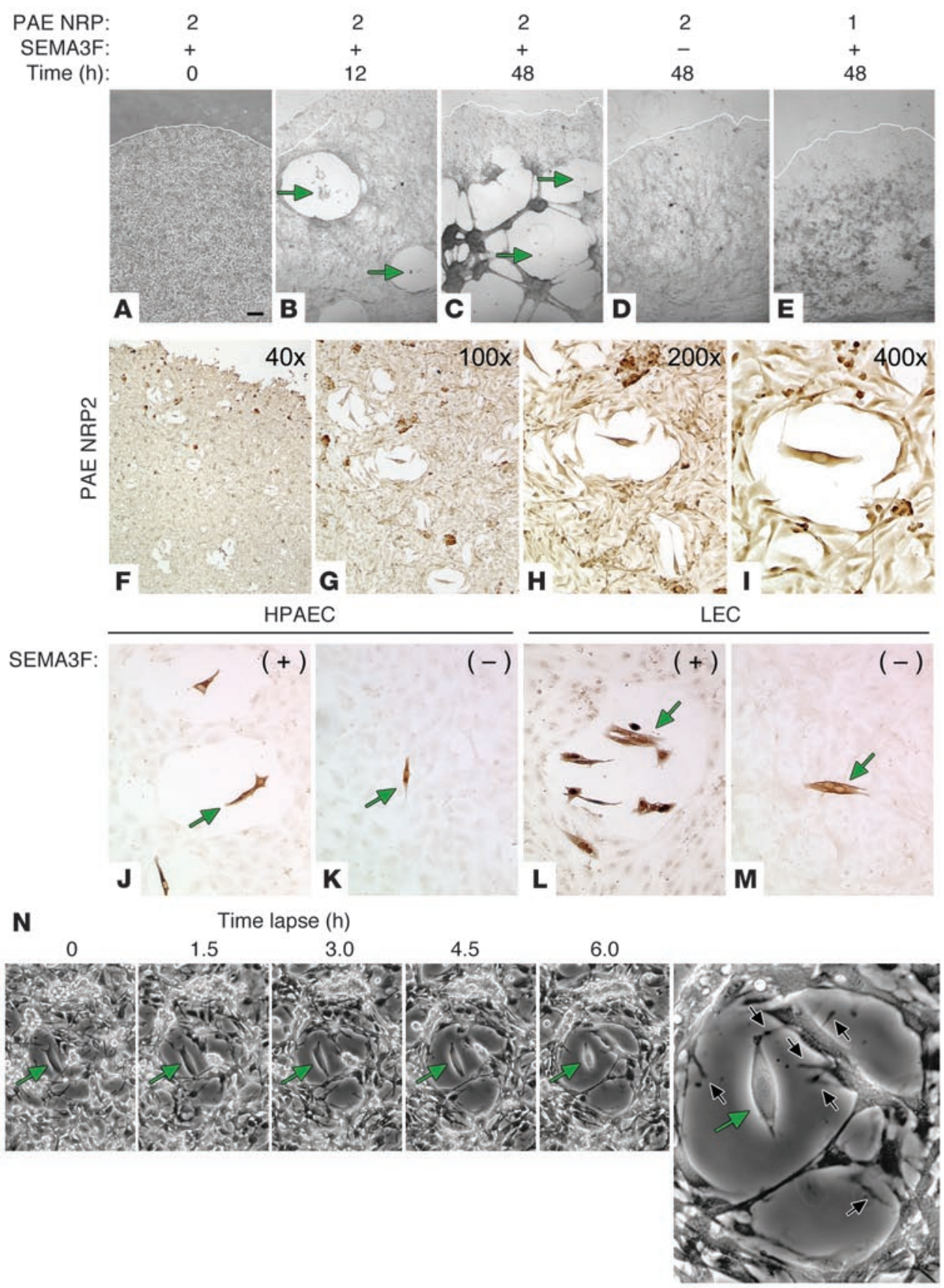

\section{Figure 7}

SEMA3F-induced EC chemorepulsion. (A-E) PAE NRP2 (A-D) or PAE NRP1 cells (E) were cultured to confluence. At time 0, SM/SEMA3F cells were added into the culture (A). EC retraction was apparent at 12 hours (B) and was even more dramatic at 48 hours (C). Green arrows point to the SM/SEMA3F cells at the center of the clearance zones. No clearance zone was observed when SM cells lacking SEMA3F were added (D) or when PAE NRP1 cells were used (E). Scale bar: $200 \mu \mathrm{m}$. (F-I) The repulsion of PAE NRP2 cells by SM/SEMA3F cells (brown color due to melanoma marker $\mathrm{S} 100 \beta$ staining) at 9 hours of coculture is shown at various magnifications $-40 \times(F)$, $100 \times(\mathbf{G}), 200 \times(\mathbf{H})$, and $400 \times(\mathbf{I})$ - to demonstrate the large number of clearance zones observable at low magnification and the clearance zone induced by 1 individual SM/SEMA3F cell at high magnification. (J-M) The repulsion of primary ECs. HPAECs (J) and LECs (L) are repelled from SM/SEMA3F cells, but HPAECs (K) and LECs (M) are not repelled from control SM/Mock cells. Representative tumor cells are denoted by green arrows. Scale bars: $100 \mu \mathrm{m}$. (N) Timelapse photography and video (see supplemental data) of the interaction of SM/SEMA3F and PAE NRP2 cells. The central tumor cell (green arrow) is expressing SEMA3F, and the surrounding cells are PAE cells expressing NRP2. Notice the ECs moving away from the source of SEMA3F over time, creating a clearance zone. In the video and in a higher-magnification view (right panel), the ECs can be seen to extend and retract numerous filopodia (black arrows) as they sense their environment. reduction in integrin levels, and in tumor cell adhesion and migration. This mechanism is consistent with previous reports that the integrin family of cell-adhesion proteins promote attachment and migration of cells on the surrounding ECM and that several integrins play key roles in promoting tumor angiogenesis and tumor metastasis (38). Another important step in metastasis is the ability of tumor cells to invade stroma. Consistent with this property, cells expressing SEMA3F exhibit decreased invasion through Matrigel-coated membranes (Matrigel, BD Biosciences) in vitro, another direct effect on tumor cells (data not shown). The significant inhibition of adhesion, migration, and invasion may explain why SM/SEMA3F cells could not metastasize even when injected directly into the circulation in the experimental-metastasis studies.
The direct effect of SEMA3F on adhesion, migration, and invasion of the SM melanoma cells is most likely mediated through NRP2, the functional SEMA3F receptor. The affinity of SEMA3F for NRP2 is 10 times greater than for NRP1 (5). SM cells express high levels of NRP2 protein, the high-affinity receptor, but undetectable levels of NRP1 protein, the low-affinity receptor. In addition, as shown by chemorepulsion experiments, SEMA3F induces repulsion only in ECs expressing NRP2, not in ECs expressing NRP1. SEMA3F also induces the repulsion of LECs, which express NRP2 only (discussed below). However, it has been reported that the effects of SEMA3F are mediated by NRP2 in C100 cells but by NRP1 in MCF7 cells (37). Thus, although it is highly unlikely, we cannot completely rule out that NRP1 is also targeted by SEMA3F. 


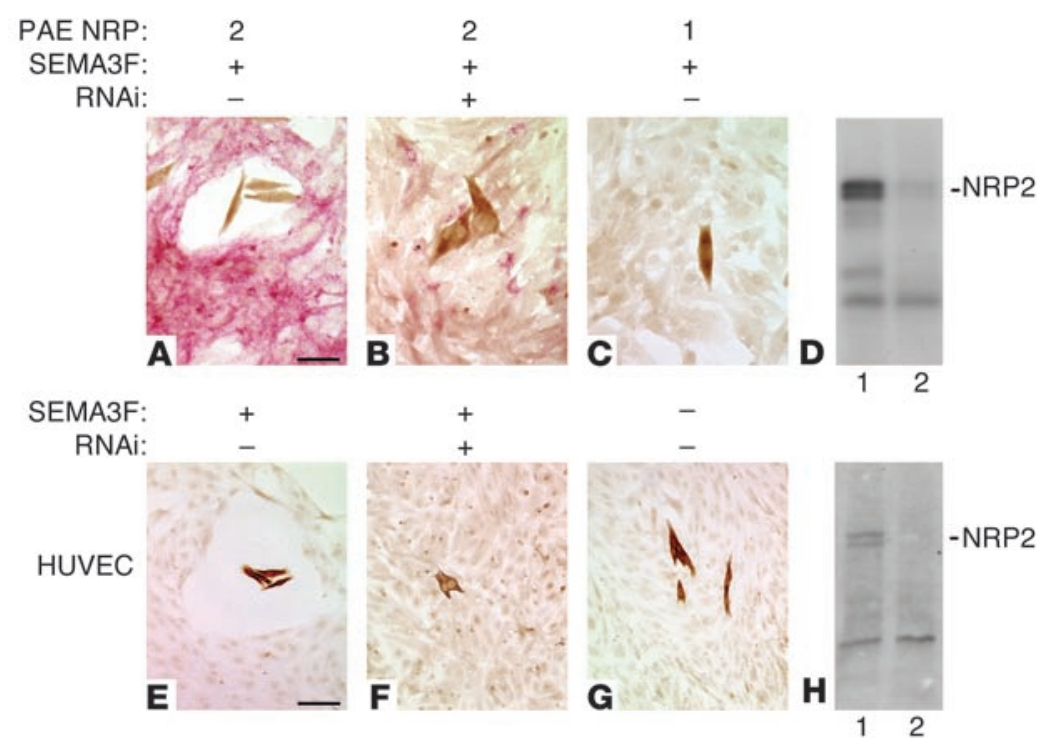

Alterations in the tumor microenvironment, resulting in tumor encapsulation, may be another mechanism contributing to SEMA3F-induced inhibition of metastasis. The tumor environment plays an important role in tumor progression. SEMA3F protein secreted by transfected SM tumors had profound effects on other cells in the tumor microenvironment. One of the most dramatic phenotypic changes in the tumors expressing SEMA3F was the level to which they were encapsulated. Control tumors had invasive leading edges that infiltrated dermal fat and muscle layers, whereas tumors expressing SEMA3F were circumscribed with thick layers of fibroblasts and collagen matrix. Encapsulation is a hallmark of benign tumor morphology and is common in lowgrade human carcinomas. The thick layer of fibroblasts, matrix, ECs, and macrophages that makes up the capsule represents a barrier that prevents the tumor cells from invading and metastasizing. Interestingly, A375P (parental) melanoma cells that are nonmetastatic and naturally express SEMA3F are also encapsulated (our unpublished data), which suggests that SEMA3F reverts aggressive tumors to their original benign state.

Neighboring epithelial cells in the tumor environment were affected as well. The epidermis overlying SM tumors was grossly hyperplastic. Epidermal hyperplasia has been associated with melanoma tumor thickness and metastasis in human patient samples as well as in several mouse models (39). The hyperplastic keratinocytes, in turn, express bFGF and VEGF $(39,40)$ that may contribute to the overall angiogenic response and eventual dissemination of tumor cells. However, in the presence of SEMA3F the epidermal cells overlying tumors remained at normal thickness. SEMA3F might interact with keratinocytes directly by binding to keratinocyte NRP1 and NRP2 or indirectly by blocking epithelial growth factors.

Inhibition of tumor angiogenesis may contribute to SEMA3F inhibition of metastasis. Previously, we showed that another semaphorin, SEMA3A, was a competitor of VEGF binding to EC NRP1, inhibited EC migration, and inhibited endothelial sprouting from an aortic ring assay (21). It seemed reasonable that the structurally related SEMA3F might have similar potential antiangiogenesis effects. After 6 weeks in nude mice, tumors expressing SEMA3F contained large areas of apoptotic cells, possibly as a result of insufficient vascularization. A statistical comparison, analyzing 100

\begin{abstract}
Figure 8
EC repulsion by SEMA3F is NRP2 dependent. (A and B) PAE NRP2 cells are stained pink for NRP2 protein. SM/SEMA3F cells (brown) induce clearance zones (A), but not in cells treated with RNAi (B). (C) No clearance zones are found in PAE NRP1 cells. (D) Western blot analysis of PAE NRP2 cells with and without RNAi. PAE NRP2/RNAi cells express dramatically reduced NRP2 protein (B; and D, lane 2). (E-H) HUVECs. SM/SEMA3F cells induce a zone of clearance $(E)$, but not in the presence of RNAi (F) or the absence of SEMA3F (G). (H) Western blot analysis of HUVECs with and without RNAi. HUVECs/RNAi cells express dramatically reduced NRP2 protein $(\mathbf{H}$, lane 2). Scale bars: $100 \mu \mathrm{m}$.
\end{abstract}

fields of CD31-stained cryosections each, indicated that tumors expressing SEMA3F had only one-half the vessel density and one-third the total vessel area of control SM tumors. Furthermore, ECs in dermal blood vessels surrounding the tumors sprouted toward and infiltrated control tumors, whereas the vessels surrounding tumors expressing SEMA3F rarely sprouted into the tumor tissue. In thick cryosections $(30 \mu \mathrm{m})$, there appeared to be an avascular zone or barrier surrounding the SEMA3F tumors. It was concluded that SEMA3F is a potent inhibitor of tumor angiogenesis and, therefore, metastasis. Microvessel density has been linked to metastatic potential in nearly every type of solid tumor $(41,42)$. It follows that the greater the number of vessels in a tumor, the greater is the chance of hematogenous metastasis or tumor cell escape from the primary site. Therefore, the lack of tumor vessels and decreased vessel area may contribute to the absence of metastasis seen in tumors overexpressing SEMA3F.

Different mechanisms have been reported to explain the activity of tumor angiogenesis inhibitors. For example, VEGF kinase inhibitors and soluble VEGFRs block VEGFR signaling needed for angiogenesis (43). Induction of apoptosis, inhibition of matrix metalloproteinase activity, and inhibition of integrin clustering by endostatin are also inhibitory mechanisms (44-46). Here we propose a novel mechanism, chemorepulsion of ECs. A coculture system was established with SM tumor cells expressing SEMA3F and PAE NRP1 or PAE NRP2. At a ratio of 1 tumor cell to $100 \mathrm{ECs}$, large zones of clearance formed around the tumor cells when ECs expressed NRP2, the functional SEMA3F receptor, but not when ECs expressed NRP1, the functional SEMA3A receptor. Time-lapse videos (see supplemental data) showed progressive enlargement of the clearance zones with time and showed an active process in which the ECs extended and retracted numerous filopodia. These results are consistent with a recent report that SEMA3F regulates EC morphology by decreasing the number of lamellipodia and increasing the number of filopodia (47). In addition, we have shown previously that SEMA3A depolymerized EC F-actin and induced retraction of lamellipodia (21). These studies indicated that semaphorins disorganize the EC cytoskeleton. The chemorepulsion of ECs by SEMA3F is not limited to engineered PAE NRP2 cells but occurs with nonengineered HUVECs, HPAECs, and LECs as well. Furthermore, the chemorepulsant activity of SEMA3F on ECs is abrogated by NRP2 RNAi, which shows that the repulsion is NRP2 dependent. The chemorepulsion of ECs is analogous to the chemorepulsion of DRG and SCG axons by Sema3A and Sema3F, respectively $(1,9,12)$. Together, these results suggest that com- 
mon semaphorin-mediated molecular mechanisms are operating in neurons and ECs, and that there are molecular similarities between neuronal guidance and angiogenesis.

Finally, SEMA3F inhibition of metastasis may be due in part to the ability of SEMA3F to inhibit lymphangiogenesis. Lymphatic vessels act as a conduit for the delivery of metastatic cells to lymph nodes (32). Invasive cells may migrate out of the primary tumor and enter peritumoral lymphatics, or lymphatic vessels may sprout into the primary tumor (lymphangiogenesis) (48). NRP2 is expressed on lymphatic vessels during development (8). Recently, lymphangiogenesis (as measured by lymphatic vessel endothelial hyaluoronan receptor-1 [LYVE-1] staining) has been proposed as a novel prognostic indicator for the risk of lymph node metastasis in cutaneous melanoma (49). Our results indicate that SEMA3F can repel purified LECs in vitro. Furthermore, preliminary data show that tumors expressing SEMA3F lack intratumoral lymphatic vessels as determined by LYVE-1 staining. These results suggest that SEMA3F may be a novel inhibitor of LEC migration and possibly of lymphangiogenesis as well.

The distinct signaling molecules involved in SEMA3F-mediated inhibition of migration or repulsion of ECs are unclear but may resemble those of SEMA3A in neurons. SEMA3A binds NRP1 and activates plexin A1 and the GTPases Rnd1 and RhoD $(50,51)$. The ensuing signal transduction pathway includes activation of Fyn/Fes (plexin-associated kinase) followed by phosphorylation of collapsin response mediator proteins (CRMPs) (52). Of particular interest to us is the observation that the expression of CRMP-1, a downstream target of Sema3A, is downregulated in invasive and metastatic tumor cells (53). Overexpression of CRMP-1 in highly invasive lung cancer CL(1-5) cells results in fewer filopodia and lowered in vitro invasive activity. This result parallels our finding that SEMA3F is strongly downregulated in highly metastatic melanoma cells and that overexpression of SEMA3F inhibits metastasis. Thus, it is plausible that CRMPs are downstream from SEMA3F and play an intermediate role in the SEMA3F-mediated inhibition of metastasis.

Under current investigation is the question of SEMA3F expression in human tumor samples. Samples of human primary melanomas and metastatic melanoma lesions are being collected, albeit with difficulty in getting matched pairs. The goal is to examine SEMA3F expression by ISH in these samples.

In summary, SEMA3F is a potent inhibitor of metastasis. It may act by various mechanisms including direct inhibition of aggressive tumor cell migration, adhesion, and invasiveness; promotion of encapsulation, leading to a benign phenotype; and inhibition of tumor angiogenesis by chemorepulsion of ECs.

\section{Methods}

Cell culture. Cell lines of low and high metastatic potential, respectively, included human melanoma cells (A375P and SM) (54) and human transitional cell (bladder) carcinoma lines (253J and 253JBV) (55) provided by Isaiah J. Fidler (Anderson Cancer Center, Houston, Texas, USA); human prostate carcinoma cells (PC3M and PC3MLN4) (56) provided by Curtis Pettaway (Anderson Cancer Center); and rat prostate carcinoma cells (AT2.1 and AT6.1) (57) provided by Bruce Zetter (Children's Hospital, Boston, Massachusetts, USA). PAE cells were provided by Lena Claesson-Welsh (Uppsala University, Uppsala, Sweden). PAE NRP1 cells were described previously (2). HPAECs and HUVECs were purchased from Cambrex Bio Science Walkersville Inc.

Human LECs. Freshly excised human foreskins were sterilized in betadine, minced, and digested in type IA-S collagenase (400 U/ml; Sigma-Aldrich).
After incubation, the enzyme was neutralized with $10 \%$ heat-inactivated FCS (HyClone) in DMEM (Invitrogen Corp.), the foreskin fragments were allowed to settle, and the cocktail supernatant was removed. A Teflon pestle (DuPont) was used to extrude cells from the digested tissue fragments. The homogenate was strained $(100 \mu \mathrm{m})$, centrifuged, and resuspended in PBS/0.1\% BSA. Cells were selected with magnetic beads coated with antihuman VEGFR-3 antibody, according to the manufacturer's instructions (Dynal Biotech), and plated on 1.5\% gelatin-coated dishes in endothelial basal medium (EBM-2; Cambrex Bio Science Walkersville Inc.), 20\% human serum (Irvine Scientific), 30\% sarcoma 180-conditioned medium, $10 \mathrm{ng} /$ $\mathrm{ml} \mathrm{FGF-2} \mathrm{(a} \mathrm{generous} \mathrm{gift} \mathrm{from} \mathrm{Scios} \mathrm{Inc.),} \mathrm{and} 10 \mu \mathrm{g} / \mathrm{ml}$ heparin (SigmaAldrich). The day after plating, colonies of 3-10 ECs were isolated with cloning cylinders (Sigma-Aldrich) and expanded. LECs were confirmed using FACS with lymphatic-specific antibodies (58).

Mice. Male, pathogen-free nude mice were purchased from Charles River Laboratories Inc. and used when they were 8-10 weeks of age. The mice were maintained under specific pathogen-free conditions in a facility accredited by the Association for Assessment and Accreditation of Laboratory Animal Care (AAALAC). The care and experimental procedures were in accordance with current regulations of the US Department of Agriculture, Department of Health and Human Services, and NIH, and the Institutional Animal Care and Use Committee at Children's Hospital.

Tumor cell inoculation. SM cells were free of mycoplasma, reovirus type 3 , pneumonia virus of mice, mouse adenovirus, murine hepatitis virus, lymphocytic choriomeningitis virus, ectromelia virus, and lactate dehydrogenase virus (Microbiological Associates). Tumor cells $\left(10^{6}\right.$ cells per $100 \mu \mathrm{l}$ of HBSS) were injected subdermally (59) into the right dorsolateral flank of nude mice. In protocol 1, mice were sacrificed 6 weeks after tumor cell inoculation. In protocol 2, primary tumors were surgically removed when the tumor size reached an average diameter of $1 \mathrm{~cm}$ (4-5 weeks after injection). Subsequently, mice were killed 12 weeks after the tumor cell injection. For experimental metastasis, mice were injected i.v. with $2 \times 10^{5}$ cells per $100 \mu \mathrm{l}$ of HBSS, and lungs were examined 12 weeks later.

Transfection. The full-length, myc-tagged, human SEMA3F construct in the pSecTag vector (Invitrogen Corp.) was provided by Marc Tessier-Lavigne (UCSF, San Francisco, California, USA). Cells were transfected with LipofectAMINE 2000 (Invitrogen Corp.) according to the manufacturer's instructions. A number of stable lines were generated, including clones A2, A3, B2, C3, and D1 that express SEMA3F, clone H10 that was transfected but does not express SEMA3F, and mock cells that were transfected with empty pSecTag vector. The full-length human NRP2 construct in the pcDNA 3.1(+) vector (Invitrogen Corp.) was provided by Seiji Takashima (Osaka University Graduate School of Medicine, Osaka, Japan). PAE cells were stably transfected with human NRP2, and expression was confirmed by Western blot analysis.

Antibodies for Western blot analysis. Anti-C-Myc mAb 9E10, anti-human NRP2 C9 mAb, and rabbit anti-human NRP1 H286 were purchased from Santa Cruz Biotechnology Inc. Anti-human $\beta 1 \mathrm{mAb} 2251$, anti-human $\alpha 5 \beta 1 \mathrm{mAb} 1950$, and rabbit anti- $\alpha 5$ polyclonal antibody 1949 were purchased from Chemicon International Inc. HRP-conjugated sheep antimouse IgG whole antibody was purchased from Amersham Biosciences.

Antibodies for IHC. Rat anti-mouse CD31 antibody was purchased from Pharmingen. Affinity-purified, mouse-absorbed, biotinylated rabbit antirat IgG $(\mathrm{H}+\mathrm{L})$ antibody was purchased from Vector Laboratories Inc. Alexa Fluor 488-conjugated goat anti-rat was purchased from Invitrogen Corp. Rabbit anti-S100 $\beta$ antibody and mouse anti-PCNA (clone PC10) mAb were purchased from DakoCytomation. Peroxidase-conjugated goat anti-rabbit $\operatorname{IgG~} \mathrm{F}\left(\mathrm{ab}^{\prime}\right)_{2}$ antibody was purchased from Jackson ImmunoResearch Laboratories Inc. Rat anti-mouse Fibroblast (Pan reticular) antibody was purchased from DPC Biermann GmbH. Rabbit anti-GFP antibody was 
purchased from Abcam Ltd. Peroxidase-conjugated rat anti-mouse IgG2a was purchased from Serotec Inc.

Antibody for ISH. Alkaline phosphatase-conjugated anti-digoxigenin Fab fragment antibody was purchased from Roche Diagnostics Corp.

Western blot analysis. Protein lysates or conditioned media were immunoprecipitated with $\alpha 5 \beta 1$ antibodies and protein G-Sepharose (Amersham Biosciences) or precipitated with Con A Sepharose (Amersham Biosciences) alone. Western blots were performed as described previously (3).

IHC. Formalin-fixed, paraffin-embedded tissues were used for routine histology (H\&E) and to identify PCNA-, GFP-, and S100ß-positive cells. Cryosections were used for H\&E, Gomori trichrome, and IHC to identify ECs (CD31) and fibroblasts. IHC was performed as described previously (40).

ISH. An antisense, human SEMA3F-specific riboprobe was produced from a cDNA containing a sequence made with primers (forward, 5 '-GAATGCGTGCTCTCAGGCAA-3'; reverse, 5'-CTCCGCACTGTCAGGAATGA$3^{\prime}$ ) and labeled with a digoxigenin RNA-labeling kit (Roche Diagnostics Corp.). ISH was performed as described previously $(3,60)$.

TUNEL. TUNEL staining was performed as described previously (61).

RT-PCR. RT-PCR was performed as described previously (4). The following primer pairs were used: human NRP1 (695-bp product) forward, $5^{\prime}$-TCCCGCCTGAACTACCCTGAA-3', and reverse, $5^{\prime}$-GCCTTGCGCTTGCTGTCA TC-3'; human NRP2 (476-bp product) forward, 5'-CCCCGAACCCAACCAGAAGA-3', and reverse, 5'-GAATGCCATCCCAGATGTCCA-3'; human VEGFR-1 (498-bp product) forward, 5'-CAAGTGGCCAGAGGCATGGAG TT-3', and reverse, 5'-GATGTAGTCTTTACCATCCTGTTG-3'; human VEGFR-2 (709-bp product) forward, 5'-GAGGGCCTCTCATGGTGATTGT-3', and reverse, 5'-TGCCAGCAGTCCAGCATGGTCTG-3'; and human GAPDH (715-bp product) forward, 5'-CAAATTCCATGGCACCGTCA-3', and reverse, 5'-GGAGTGGGTGTCGCTGTTGA-3'.

Northern blot analysis. Polyadenylated mRNA preparation, RNA gel electrophoresis, blotting, and hybridization were performed as described previously (3). Probes were made from PCR products corresponding to human NRP1 b domain (951 bp): forward, $5^{\prime}$-GAAGATTTCAAATGTATGGAAG$3^{\prime}$, and reverse, $5^{\prime}$-GGCTTCCACTTCACAGCCCAG-3'; to human NRP2 b domain (618 bp): forward, 5'-CTGCTGGGTCCTGGGGTCC-3', and reverse, 5'-ACGGCCATCGCAACACAGG-3'; to human SEMA3F (408 bp): forward, 5'-GAATGCGTGCTCTCAGGCAA-3', and reverse, 5'-CTCCGCACTGTCAGGAATGA-3'; and to actin (184 bp): forward, $5^{\prime}$-ATGCCCCCCGTGCTGTGTTCC-3', and reverse, 5'-CCAGATCTTCTCCATATCATC-3'

Quantification of blood vessel staining. Five mice per group were injected with mock-transfected or SEMA3F-transfected cells, and tumors were removed after 25 days. Cryosections were stained with CD31, and digital images were obtained from 20 fields per tumor, which covers the entire cross-section. Morphometric analysis was performed on 100 separate digital images per group using the IPLab software (Scanalytics Inc.).

ELISA. SM cells $\left(10^{6}\right)$ were cultured overnight in $5 \mathrm{ml}$ of serum-free MEM. Conditioned medium was analyzed using the human VEGF Quantikine Immunoassay (R\&D Systems Inc.) according to the manufacturer's instructions.

Adhesion assay. Tumor cells ( $10^{5}$ cells per well) in MEM/ $1 \%$ BSA were seeded in nonadhesive Falcon 24-well culture plates (BD Biosciences - Discovery Labware) coated with increasing concentrations of FN (BD Biosciences) for 30 minutes at $37^{\circ} \mathrm{C}$. The cells were washed 3 times with warm PBS, and remaining adherent cells were trypsinized and counted in a Coulter Counter (Beckman Coulter Inc.). The results represent the average of 3 wells per cell line. The experiment was repeated twice with similar results.
Motility assay. Basal tumor cell motility was measured in a Boyden chamber as described previously with modifications (21). Tumor cells (15,000 cells per well) in MEM/0.1\% BSA were added in the upper chambers, and medium containing $0.1 \% \mathrm{FBS}$ was added in the lower chambers. After 5 hours at $37^{\circ} \mathrm{C}$, the number of cells migrating through a $12-\mu \mathrm{m}, \mathrm{FN}$-coated Poretics polycarbonate membrane (GE Osmonics) was counted. The results represent the average of 4 wells per cell line. The experiment was repeated 3 times with similar results.

Proliferation assay. Tumor cell proliferation was measured as described previously (62). The results (cpm) represent the average of 12 wells per cell line. The experiment was repeated twice with similar results.

RNAi. siGENOME SMARTpool NRP2 (M-017721) was purchased from Dharmacon and used according to the manufacturer's instructions.

Chemorepulsion assay. One EC line (PAE NRP1, PAE NRP2, HPAECs, HUVECs, or LECs) and one tumor cell line (SM/Mock or SM/SEMA3F) were each plated confluently into separate $5-\mathrm{mm}$ cloning tubes on glass slides (Fisher Scientific International Co.) or in $35-\mathrm{mm}$ plastic dishes (BD Biosciences - Discovery Labware) overnight in complete media. The following day, the dish or slide was washed, and the cloning tubes were removed to reveal 2 circles of confluent cells and many floating tumor cells that were free to adhere within the EC monolayer. The medium was changed to serum-free F-12 Nutrient Mixture (Ham) (Invitrogen Corp.) for porcine ECs or serum-free endothelial basal medium (EBM-2) for human ECs, and cocultures were incubated for various times. Alternatively, ECs and tumor cells were premixed at a ratio of 100:1 in F-12 media and seeded confluently onto slides or plastic dishes. Cells were photographed using an Eclipse TE300 microscope (Nikon Inc.) and a SPOT RT camera (Diagnostic Instruments Inc.). For time-lapse photography, images were captured every 3 minutes for 9 hours using SPOT 3.2.4 software (Diagnostic Instruments Inc.) on a temperature-controlled stage with HEPES buffer ( $\mathrm{pH} 7.2)$ in the media.

\section{Acknowledgments}

This work was supported by NIH grants CA37392 and CA45548 and the Harvard Skin Disease Research Center (to M. Klagsbrun); the Elizabeth and George Sanborn Foundation Fellowship through the American Cancer Society (to D. Bielenberg); the Uehara Memorial Foundation Fellowship, Japan (to Y. Hida); the Dr. Mildred Scheel Cancer Foundation Fellowship, Germany (to M. Kreuter); and the Howard Hughes Medical Institute Research Fellowship Award (to C. Choi Kim). We thank Isaiah Fidler, Curtis Pettaway, Bruce Zetter, and Lena Claesson-Welsh for cell lines. We thank Marc Tessier-Lavigne and Seiji Takashima for constructs. We thank Judah Folkman, Bruce Zetter, Roni Mamluk, Dipak Panigraphy, Jacqueline Banyard, Sarah Short, and Kyoko Hida for thoughtful discussions and reading of the manuscript. We thank Kristin Gullage, Melissa Mang, Sandra Smith, Emy Chen, and Ricardo Sanchez for technical assistance.

Received for publication February 19, 2004, and accepted in revised form September 14, 2004.

Address correspondence to: Michael Klagsbrun, Department of Pathology, Children's Hospital, Harvard Medical School, 300 Longwood Avenue, New Research Building 12210, Boston, Massachusetts 02115, USA. Phone: (617) 919-2157; Fax: (617) 730-0233; E-mail: michael.klagsbrun@childrens.harvard.edu.
1. Kolodkin, A.L., et al. 1997. Neuropilin is a semaphorin III receptor. Cell. 90:753-762.

2. Soker, S., Takashima, S., Miao, H.Q., Neufeld, G., and Klagsbrun, M. 1998. Neuropilin-1 is expressed by endothelial and tumor cells as an isoform-specific receptor for vascular endothelial growth factor. Cell. 92:735-745.

3. Gagnon, M.L., et al. 2000. Identification of a natural soluble neuropilin-1 that binds vascular endothelial growth factor: in vivo expression and antitumor activity. Proc. Natl. Acad. Sci. U. S. A. 97:2573-2578. 
4. Harper, J., Gerstenfeld, L.C., and Klagsbrun, M. 2001. Neuropilin-1 expression in osteogenic cells: down-regulation during differentiation of osteoblasts into osteocytes. J. Cell. Biochem. 81:82-92.

5. Chen, H., Chedotal, A., He, Z., Goodman, C.S., and Tessier-Lavigne, M. 1997. Neuropilin-2, a novel member of the neuropilin family, is a high affinity receptor for the semaphorins Sema E and Sema IV but not Sema III. Neuron. 19:547-559.

6. Rossignol, M., Beggs, A.H., Pierce, E.A., and Klagsbrun, M. 1999. Human neuropilin-1 and neuropilin2 map to $10 \mathrm{p} 12$ and $2 \mathrm{q} 34$, respectively. Genomics. 57:459-460.

7. Herzog, Y., Kalcheim, C., Kahane, N., Reshef, R., and Neufeld, G. 2001. Differential expression of neuropilin-1 and neuropilin-2 in arteries and veins. Mech. Dev. 109:115-119.

8. Yuan, L., et al. 2002. Abnormal lymphatic vessel development in neuropilin 2 mutant mice. Development. 129:4797-4806.

9. Chen, H., He, Z., Bagri, A., and Tessier-Lavigne, M. 1998. Semaphorin-neuropilin interactions underlying sympathetic axon responses to class III semaphorins. Neuron. 21:1283-1290.

10. Chedotal, A., et al. 1998. Semaphorins III and IV repel hippocampal axons via two distinct receptors. Development. 125:4313-4323.

11. He, Z., and Tessier-Lavigne, M. 1997. Neuropilin is a receptor for the axonal chemorepellent Semaphorin III. Cell. 90:739-751.

12. Giger, R.J., et al. 1998. Neuropilin-2 is a receptor for semaphorin IV: insight into the structural basis of receptor function and specificity. Neuron. 21:1079-1092.

13. Klagsbrun, M., Takashima, S., and Mamluk, R. 2002. The role of neuropilin in vascular and tumor biology. Adv. Exp. Med. Biol. 515:33-48.

14. Takahashi, T., et al. 1999. Plexin-neuropilin-1 complexes form functional semaphorin-3A receptors. Cell. 99:59-69.

15. Soker, S., Miao, H.Q., Nomi, M., Takashima, S., and Klagsbrun, M. 2002. VEGF165 mediates formation of complexes containing VEGFR-2 and neuropilin1 that enhance VEGF165-receptor binding. J. Cell. Biochem. 85:357-368.

16. Kitsukawa, T., et al. 1997. Neuropilin-semaphorin III/D-mediated chemorepulsive signals play a crucial role in peripheral nerve projection in mice. Neuron. 19:995-1005.

17. Takashima, S., et al. 2002. Targeting of both mouse neuropilin- 1 and neuropilin- 2 genes severely impairs developmental yolk sac and embryonic angiogenesis. Proc. Natl. Acad. Sci. U. S. A. 99:3657-3662.

18. Lee, P., et al. 2002. Neuropilin-1 is required for vascular development and is a mediator of VEGFdependent angiogenesis in zebrafish. Proc. Natl. Acad. Sci. U. S. A. 99:10470-10475.

19. Miao, H.Q., Lee, P., Lin, H., Soker, S., and Klagsbrun, M. 2000. Neuropilin-1 expression by tumor cells promotes tumor angiogenesis and progression. FASEB J. 14:2532-2539.

20. Parikh, A.A., et al. 2004. Neuropilin-1 in human colon cancer: expression, regulation, and role in induction of angiogenesis. Am. J. Pathol. 164:2139-2151.

21. Miao, H.Q., et al. 1999. Neuropilin-1 mediates collapsin-1/semaphorin III inhibition of endothelial cell motility: functional competition of collapsin-1 and vascular endothelial growth factor-165. J. Cell Biol. 146:233-242.

22. Serini, G., et al. 2003. Class 3 semaphorins control vascular morphogenesis by inhibiting integrin function. Nature. 424:391-397.

23. Bates, D., et al. 2003. Neurovascular congruence results from a shared patterning mechanism that utilizes Semaphorin3A and Neuropilin-1. Dev. Biol. 255:77-98.
24. Kessler, O., et al. 2004. Semaphorin-3F is an inhibitor of tumor angiogenesis. Cancer Res. 64:1008-1015.

25. Sekido, Y., et al. 1996. Human semaphorins A(V) and IV reside in the 3 p21.3 small cell lung cancer deletion region and demonstrate distinct expression patterns. Proc. Natl. Acad. Sci. U. S. A. 93:4120-4125.

26. Roche, J., et al. 1996. Distinct 3p21.3 deletions in lung cancer and identification of a new human semaphorin. Oncogene. 12:1289-1297.

27. Tomizawa, Y., et al. 2001. Inhibition of lung cancer cell growth and induction of apoptosis after reexpression of $3 \mathrm{p} 21.3$ candidate tumor suppressor gene SEMA3B. Proc. Natl. Acad. Sci. U. S. A. 98:13954-13959.

28. Tse, C., Xiang, R.H., Bracht, T., and Naylor, S.L. 2002. Human Semaphorin 3B (SEMA3B) located at chromosome 3 p21.3 suppresses tumor formation in an adenocarcinoma cell line. Cancer Res. 62:542-546.

29. Xiang, R., et al. 2002. Semaphorin 3 F gene from human 3 p 21.3 suppresses tumor formation in nude mice. Cancer Res. 62:2637-2643.

30. McCarty, M.F., Bielenberg, D., Donawho, C., Bucana, C.D., and Fidler, I.J. 2002. Evidence for the causal role of endogenous interferon-alpha/beta in the regulation of angiogenesis, tumorigenicity, and metastasis of cutaneous neoplasms. Clin. Exp. Metastasis. 19:609-615.

31. Fidler, I.J. 1996. Critical determinants of melanoma metastasis. J. Investig. Dermatol. Symp. Proc. 1:203-208.

32. Detmar, M., and Hirakawa, S. 2002. The formation of lymphatic vessels and its importance in the setting of malignancy. J. Exp. Med. 196:713-718.

33. Hong, Y.K., et al. 2002. Prox1 is a master control gene in the program specifying lymphatic endothelial cell fate. Dev. Dyn. 225:351-357.

34. Xiang, R.H., et al. 1996. Isolation of the human semaphorin III/F gene (SEMA3F) at chromosome 3 p21, a region deleted in lung cancer. Genomics. 32:39-48.

35. Clarkson, K.S., Sturdgess, I.C., and Molyneux, A.J. 2001. The usefulness of tyrosinase in the immunohistochemical assessment of melanocytic lesions: a comparison of the novel T311 antibody (anti-tyrosinase) with S-100, HMB45, and A103 (anti-melan-A). J. Clin. Pathol. 54:196-200.

36. Fidler, I.J. 1990. Critical factors in the biology of human cancer metastasis: twenty-eighth G.H.A. Clowes memorial award lecture. Cancer Res. 50:6130-6138.

37. Nasarre, P., et al. 2003. Semaphorin SEMA3F and VEGF have opposing effects on cell attachment and spreading. Neoplasia. 5:83-92.

38. Jin, H., and Varner, J. 2004. Integrins: roles in cancer development and as treatment targets. Br.J. Cancer. 90:561-565.

39. McCarty, M.F., et al. 2003. Epidermal hyperplasia overlying human melanoma correlates with tumour depth and angiogenesis. Melanoma Res. 13:379-387.

40. Bielenberg, D.R., et al. 1998. Molecular regulation of UVB-induced cutaneous angiogenesis. J. Invest. Dermatol. 111:864-872.

41. Hasan, J., Byers, R., and Jayson, G.C. 2002. Intratumoural microvessel density in human solid tumours. Br. J. Cancer. 86:1566-1577.

42. Srivastava, A., Laidler, P., Davies, R.P., Horgan, K., and Hughes, L.E. 1988. The prognostic significance of tumor vascularity in intermediate-thickness (0.76-4.0 mm thick) skin melanoma. A quantitative histologic study. Am. J. Pathol. 133:419-423.

43. Ferrara, N., Gerber, H.P., and LeCouter, J. 2003. The biology of VEGF and its receptors. Nat. Med. 9:669-676.

44. Rehn, M., et al. 2001. Interaction of endostatin with integrins implicated in angiogenesis. Proc.
Natl. Acad. Sci. U. S. A. 98:1024-1029.

45. Kim, Y.M., et al. 2000. Endostatin inhibits endothelial and tumor cellular invasion by blocking the activation and catalytic activity of matrix metalloproteinase. Cancer Res. 60:5410-5413.

46. Dhanabal, M., et al. 1999. Endostatin induces endothelial cell apoptosis. J. Biol. Chem. 274:11721-11726.

47. Kusy, S., et al. 2003. Redundant functions but temporal and regional regulation of two alternatively spliced isoforms of Semaphorin $3 \mathrm{~F}$ in the nervous system. Mol. Cell. Neurosci. 24:409-418.

48. Stacker, S.A., Achen, M.G., Jussila, L., Baldwin, M.E., and Alitalo, K. 2002. Lymphangiogenesis and cancer metastasis. Nat. Rev. Cancer. 2:573-583.

49. Dadras, S.S., et al. 2003. Tumor lymphangiogenesis: a novel prognostic indicator for cutaneous melanoma metastasis and survival. Am. J. Pathol. 162:1951-1960.

50. Rohm, B., Ottemeyer, A., Lohrum, M., and Puschel, A.W. 2000. Plexin/neuropilin complexes mediate repulsion by the axonal guidance signal semaphorin 3A. Mech. Dev. 93:95-104.

51. Zanata, S.M., Hovatta, I., Rohm, B., and Puschel, A.W. 2002. Antagonistic effects of Rnd 1 and RhoD GTPases regulate receptor activity in Semaphorin 3A-induced cytoskeletal collapse. J. Neurosci. 22:471-477.

52. Mitsui, N., et al. 2002. Involvement of Fes/Fps tyrosine kinase in semaphorin $3 \mathrm{~A}$ signaling. $E M B O$ J. 21:3274-3285.

53. Shih, J.Y., et al. 2001. Collapsin response mediator protein-1 and the invasion and metastasis of cancer cells. J. Natl. Cancer Inst. 93:1392-1400.

54. Kozlowski, J.M., Hart, I.R., Fidler, I.J., and Hanna, N. 1984. A human melanoma line heterogeneous with respect to metastatic capacity in athymic nude mice. J. Natl. Cancer Inst. 72:913-917.

55. Dinney, C.P., et al. 1995. Isolation and characterization of metastatic variants from human transitional cell carcinoma passaged by orthotopic implantation in athymic nude mice. J. Urol. 154:1532-1538.

56. Pettaway, C.A., et al. 1996. Selection of highly metastatic variants of different human prostatic carcinomas using orthotopic implantation in nude mice. Clin. Cancer Res. 2:1627-1636.

57. Isaacs, J.T., Isaacs, W.B., Feitz, W.F., and Scheres, J. 1986. Establishment and characterization of seven Dunning rat prostatic cancer cell lines and their use in developing methods for predicting metastatic abilities of prostatic cancers. Prostate. 9:261-281.

58. Jussila, L., et al. 1998. Lymphatic endothelium and Kaposi's sarcoma spindle cells detected by antibodies against the vascular endothelial growth factor receptor-3. Cancer Res. 58:1599-1604.

59. Cornil, I., Man, S., Fernandez, B., and Kerbel, R. 1989. Enhanced tumorigenicity, melanogenesis, and metastases of a human malignant melanoma after implantation in nude mice. J. Natl. Cancer Inst. 81:938-944.

60. Komminoth, P. 2002. Detection of mRNA in tissue sections using DIG-labeled RNA and oligonucleotide probes. In Nonradioactive in situ bybridization application manual. D. Eisel, S. Grunewald-Janho, and B. Kruchen, editors. Roche Applied Science. Indianapolis, Indiana, USA. 149-163.

61. Dong, Z., et al. 1999. Suppression of angiogenesis, tumorigenicity, and metastasis by human prostate cancer cells engineered to produce interferon-beta. Cancer Res. 59:872-879.

62. Soker, S., Gollamudi-Payne, S., Fidder, H., Charmahelli, H., and Klagsbrun, M. 1997. Inhibition of vascular endothelial growth factor (VEGF)induced endothelial cell proliferation by a peptide corresponding to the exon 7-encoded domain of VEGF165. J. Biol. Chem. 272:31582-31588. 